\title{
Tight Wavelet Frame Decomposition and Its Application in Image Processing
}

\author{
Mahmud Yunus ${ }^{1,2}$ \& Hendra Gunawan ${ }^{1}$ \\ ${ }^{1}$ Analysis and Geometry Group, FMIPA ITB, Bandung \\ ${ }^{2}$ Department of Mathematics, FMIPA ITS, Surabaya
}

\begin{abstract}
This paper is devoted to the formulation of a decomposition algorithm using tight wavelet frames, in a multivariate setting. We provide an alternative method for decomposing multivariate functions without accomplishing any tensor product. Furthermore, we give explicit examples of its application in image processing, particularly in edge detection and image denoising. Based on our numerical experiment, we show that the edge detection and the image denoising methods exploiting tight wavelet frame decomposition give better results compare with the other methods provided by MATLAB Image Processing Toolbox and classical wavelet methods.
\end{abstract}

Keywords: decomposition; edge detection; frame; image denoising; multiresolution analysis; wavelet.

\section{Introduction}

In this paper, we provide an alternative method for decomposing functions in a multivariate setting. Extension from univariate function space to the multivariate setting can be accomplished trivially by considering the space generated by tensor product of univariate basis functions. Here, we propose the method by using tight wavelet frames, instead of accomplishing a tensor product.

Before going further to the main discussion, let us introduce some notations used in this paper. For $\mathbf{Z}^{d}$ be a $d$-copy of the set of integers and a finite subset $\Psi$ of $L^{2}\left(\mathbf{R}^{d}\right)$, let

$$
\Lambda(\Psi):=\left\{\psi_{j, k}: \psi \in \Psi, j \in \mathbf{Z}, k \in \mathbf{Z}^{d}\right\},
$$

where for all $j \in \mathbf{Z}$ and $j \in \mathbf{Z}^{d}$ we write $\psi_{j, k}:=2^{j d / 2} \psi\left(2^{j} \cdot-k\right)$. We say that $\Lambda(\Psi)$ is a frame if there exist two positive numbers $A$ and $B$ such that

$$
A\|f\|^{2} \leq \sum_{g \in \Lambda(\Psi)}|\langle f, g\rangle|^{2} \leq B\|f\|^{2}
$$

Received January $21^{\text {st }}, 2008$, Revised July $4^{\text {th }}, 2008$, Accepted for publication July $14^{\text {th }}, 2008$. 
for all $f \in L^{2}\left(\mathbf{R}^{d}\right)$. If $A=B$, then $\Lambda(\Psi)$ is a tight frame [1]. Such frames, which in Hilbert spaces are recognized as a generalization of orthonormal bases, play a fundamental role in signal processing, image processing, data compression and transmission, sampling theory and more, as well as being an interesting subject of research in modern and applied mathematics.

Ron and Shen [2] introduced the construction of tight wavelet frame from multi resolution analysis (MRA). We know that in applications one uses tight wavelet frames due to their efficiencies. Tight wavelet frames are efficient tools for separating several high-pass frequency parts from low-pass frequency parts. Also, frame redundancy is known to be useful for recovering information from the corrupted one.

In this paper, we follow the notion of MRA for wavelet in [2] which is slightly a modified version of the classical definition of MRA. Let $\phi \in L^{2}\left(\mathbf{R}^{d}\right)$ be a compactly supported function satisfying the following conditions:

(i) There exists a trigonometric polynomial $H$ in $e^{i \xi}$ such that

$$
\widehat{\phi}(\xi)=H(\xi / 2) \hat{\phi}(\xi / 2)
$$

If we write $H(\xi):=2^{-d} \sum_{k \in \mathbf{Z}^{d}} h_{k} e^{i \xi \cdot k}$ for a sequence $\left\{h_{k}\right\}$ in $\ell^{2}\left(\mathbf{R}^{d}\right)$, then

$$
\phi=\sum_{k \in \mathbf{Z}^{d}} h_{k} \phi(2 \cdot-k) .
$$

(ii) $\lim _{\xi \rightarrow 0} \widehat{\phi}(\xi)=1$.

Next, we denote for all $j \in Z$

$$
V_{j}:=\overline{\operatorname{span}}\left\{2^{j d / 2} \phi\left(2^{j} \cdot-k\right): k \in \mathbf{Z}^{d}\right\} .
$$

Then $V_{j}$ satisfy all conditions in classical definition of MRA [3] except that $\phi$ and its shifts constitute a Riesz (or orthonormal) basis of $V_{0}$. In this definition, the function $\phi$ is familiarly known as the scaling function for the MRA $\left\{V_{j}\right\}$.

Now, let $\phi \in L^{2}\left(\boldsymbol{R}^{d}\right)$ be a compactly supported function which satisfies the refinement equation (1). We call $H$ in (1) a filter function and $\left\{h_{k}\right\}$ a filter (sequence) associated with $\phi$. The well known method for constructing 
wavelet frame is the following condition called Unitary Extension Principle (UEP) developed by Ron and Shen in [2]:

$$
H(\xi) \overline{H(\xi+\eta)}+\sum_{\mu=1}^{r} G^{\mu}(\xi) \overline{G^{\mu}(\xi+\eta)}=\left\{\begin{array}{lll}
1, & \text { if } & \eta=0 \\
0, & \text { if } & \eta \in\{0, \pi\}^{d} \backslash\{0\}
\end{array}\right.
$$

In this case, we have to find trigonometric functions $G^{\mu}$ 's and wavelet frame generators $\psi^{\mu}$,s defined by

$$
\widehat{\psi}^{\mu}(\xi):=G^{\mu}(\xi / 2) \widehat{\phi}(\xi / 2), \quad \mu=1, \ldots, r .
$$

As shown in [4], there are sequences $\left\{g_{k}^{\mu}\right\}$ in $\ell^{2}, \mu=1, \ldots, r$, and if we write $G^{\mu}=2^{-d} \sum g_{k}^{\mu} e^{i \xi \cdot k}$ then we have

$$
\psi^{\mu}=\sum_{k \in \mathbf{Z}^{d}} g_{k}^{\mu} \phi(2 \cdot-k)
$$

We call $G^{\mu}$ as the filter function associated with $\psi^{\mu}$ and $\left\{g_{k}^{\mu}\right\}$ as its corresponding filter (sequence).

Throughout this paper we work with wavelet frames constructed by using UEP of Ron and Shen's as above. In this case, we implement a fast algorithm of decomposition and reconstruction of function by using compactly supported tight wavelet frame related to an MRA. In particular, we apply the wavelet frames constructed by Lai and Stöckler [4] and Skopina [5] in image processing.

The rest of this paper is organized as follows. In Section 2, we briefly introduce the wavelet frame decomposition and reconstruction, as well as define the algorithm. In Section 3, we implement the algorithm developed in Section 2 and adapt it to edge detection and image denoising processes. In addition, the numerical results are presented in Section 3 and the conclusions are given in Section 4.

\section{Wavelet Frame Decomposition and Reconstruction}

Suppose that $\phi$ is a scaling function for MRA $\left\{V_{j}\right\}$ with filter sequence $\left\{h_{k}\right\}$ and $\phi$ has an associated wavelet frame generator $\Psi=\left\{\psi^{1}, \ldots, \psi^{r}\right\}$ with filter sequences $\left\{g_{k}^{\mu}\right\}$, for $\mu=1, \ldots, r$. 
For all $j \in \mathbf{Z}$ and $k \in \mathbf{Z}^{d}$, we denote

$$
f_{j, k}=2^{j d / 2} f\left(2^{j} \cdot-k\right)
$$

so that we can write, for each $j \in \mathbf{Z}$,

$$
V_{j}=\overline{\operatorname{span}}\left\{\phi_{j, k}: k \in \mathbf{Z}^{d}\right\},
$$

Now, by setting

$$
W_{j}=\overline{\operatorname{span}}\left\{\psi_{j, k}^{\mu}: \mu=1, \ldots, r, k \in \mathbf{Z}^{d}\right\},
$$

it follows that

$$
V_{j+1}=V_{j}+W_{j}, \quad j \in \mathbf{Z}
$$

(see for example [1, 3, 6, 7] for the detail of this decomposition). Note that decomposition (9) is not a direct sum decomposition since in general $V_{j} \cap W_{j} \neq\{0\}$.

Recall that, in fact, any family $\left\{f_{i}\right\}_{: i \in I}$ is a frame for $\overline{\operatorname{span}}\left\{f_{i}: i \in I\right\}$ (see e.g. [7, Theorem 5.1.3]). We then use this fact together with (7), (8), and (9), for any $f \in V_{j+1}$ to get

$$
f=\sum_{k \in \mathbf{Z}^{d}}\left\langle f, \phi_{j, k}\right\rangle \phi_{j, k}+\sum_{\mu=1}^{r} \sum_{k \in \mathbf{Z}^{d}}\left\langle f, \psi_{j, k}^{\mu}\right\rangle \psi_{j, k}^{\mu}
$$

Now, for any $f \in L^{2}\left(\mathbf{R}^{d}\right)$ consider

$$
c_{j, k}:=\left\langle f, \phi_{j, k}\right\rangle \text { and } d_{j, k}^{\mu}:=\left\langle f, \psi_{j, k}^{\mu}\right\rangle, \mu=\mathbf{1}, \ldots, r .
$$

For all $j \in \mathbf{Z}, m \in \mathbf{Z}^{d}$, and $\mu=1, \ldots, r$, and by two-scale relation (2) and (5) we have

$$
\phi_{j, m}=2^{-d / 2} \sum_{k \in \mathbf{Z}^{d}} h_{k-2 m} \phi_{j+1, k} \text { and } \psi_{j, m}^{\mu}=2^{-d / 2} \sum g_{k-2 m}^{\mu} \psi_{j+1, k}^{\mu}
$$

By taking the inner products with $f$ on both sides of the two equations in (12), we have a tight wavelet frame decomposition: 


$$
c_{j, m}=2^{-d / 2} \sum_{k \in \mathbf{Z}^{d}} h_{k-2 m} c_{j+1, k} \text { and } d_{j, m}^{\mu}=2^{-d / 2} \sum_{k \in \mathbf{Z}^{d}} g_{k-2 m}^{\mu} c_{j+1, k}
$$

Using the fact that $\phi_{j, k} \in V_{j}$ and two-scale relation (2) and (5), from (10) we also have

$$
\phi_{j+1, m}=2^{-d / 2} \sum_{k \in \mathbf{Z}^{d}}\left[h_{m-2 k} \phi_{j, k}+\sum_{\mu=1}^{r} g_{m-2 k}^{\mu} \psi_{j, k}^{\mu}\right]
$$

By taking inner products with $f$ on both sides of (14), we have a tight wavelet frame reconstruction:

$$
c_{j+1, m}=2^{-d / 2} \sum_{k \in \mathbf{Z}^{d}}\left[h_{m-2 k} c_{j, k}+\sum_{\mu=1}^{r} g_{m-2 k}^{\mu} d_{j, k}^{\mu}\right]
$$

Note that all sequences $\left\{h_{m-2 k}\right\}$ and $\left\{g_{m-2 k}^{\mu}\right\}$ are finite for all $m$, because $\phi_{j, k}$ and $\psi_{j, k}^{\mu}$ are compactly supported for all $j \in \mathbf{Z}, k \in \mathbf{Z}^{d}$.

We summarize and illustrate the tight wavelet frame decomposition and tight wavelet frame reconstruction in the Figure 1.

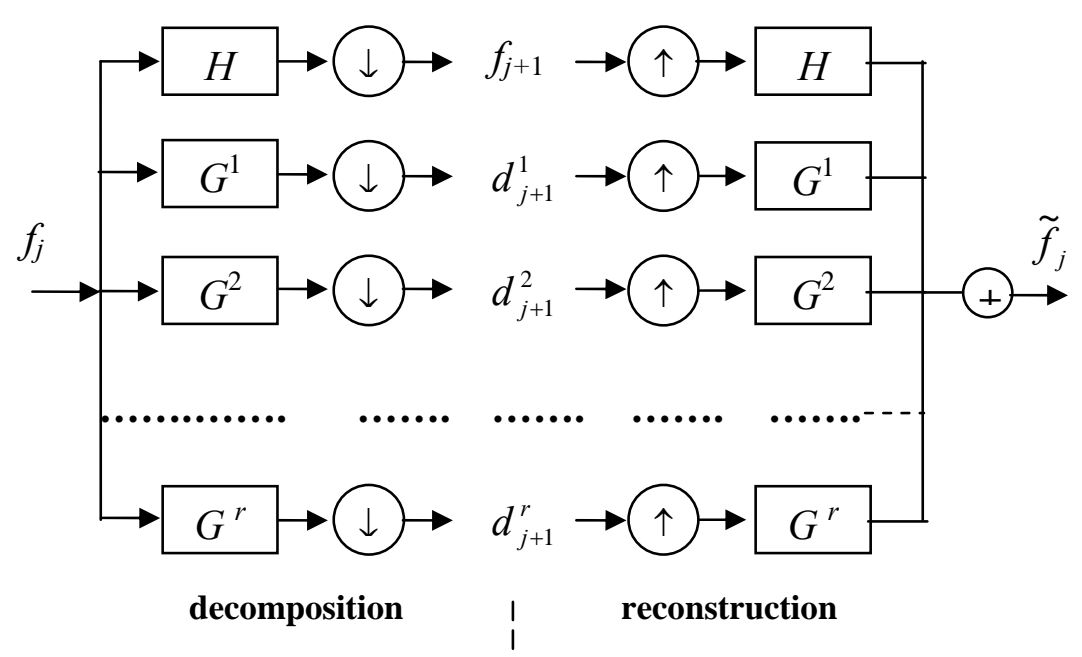

Figure 1 Tight wavelet frame decomposition and reconstruction.

It can be seen from the diagram above that in practical use of decomposition and reconstruction by using tight wavelet frame are just similar to the one using 
orthonormal wavelet basis. In this case, the computational complexity of tight wavelet frame decomposition is same as the computational complexity of orthonormal wavelet decomposition (see e.g. [3]), that is of order $O(N)$ for an input $N=M_{1} \times M_{2}$ matrix.

\section{$3 \quad$ Numerical Experiment}

We may consider the function $f$ (in a discrete form) described in Section 2, as an image (in a matrix form). The wavelet frame decomposition separates the low frequency (smooth) parts from the high frequency (detail) parts of images effectively. It is well known that all the edges and noise are related to the high frequency parts of images.

In this numerical experiment, we implement the tight wavelet frame decomposition (TWFD) and reconstruction algorithms (13) and (15) by making use of wavelet frames based on box splines in [4]. This implementation is applied for edge detection and image denoising on six test images (Figure 2).

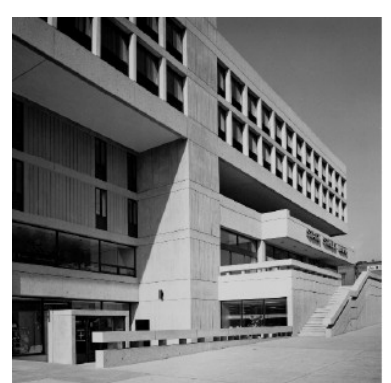

Bank

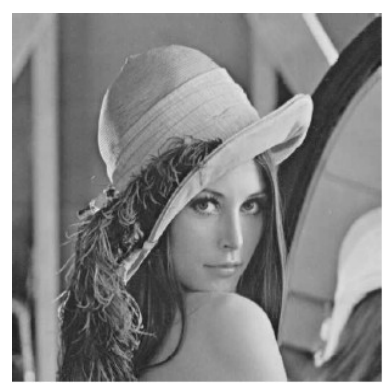

Lena

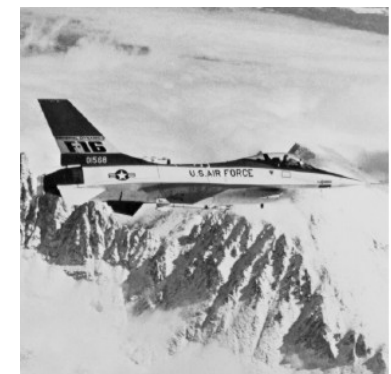

F16

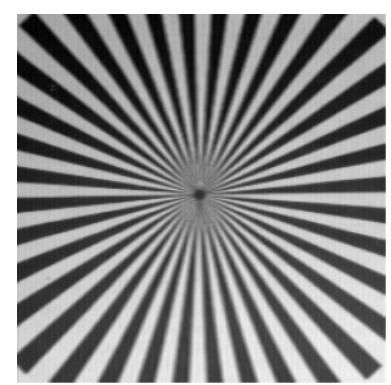

Partition

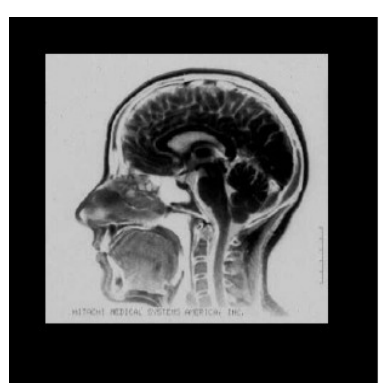

Head MRI

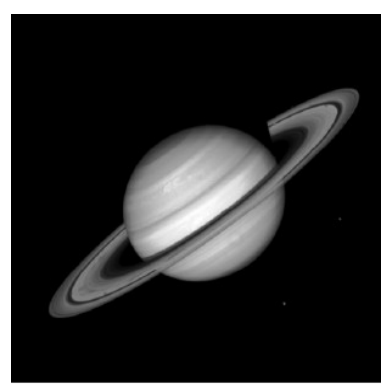

Saturn

Figure 2 Test Images. 
We choose these six different test images from MATLAB Image Processing Toolbox, by virtue of different patterns, namely: linearly edges, common long distance outdoor picture, negative film picture, common indoor (face) picture, patterned image, and curvy image.

The test image — with a gray level intensity — can be considered as a matrix with each component varies from 0 to 255 . Here, we consider 0 as black and 255 as white. Let $X_{j}:=\left[x_{k_{1}, k_{2}}^{j}\right]$ be a sub image of size $I / 2^{j} \times J / 2^{j}$, where $I$ and $J$ are usually some power of 2 and $j \in \mathbf{Z}_{+}$. We consider $X_{0}$ as the original image and $X_{j}$ as the image at the $j$ th level decomposition. We call each value $x_{k_{1}, k_{2}}^{j}$ as a pixel value at each $j$ th level. Let $H$ be a matrix whose components are obtained by the finite sequence $\left\{h_{k_{1}, k_{2}}\right\}$ satisfying two-scale relation of the compactly supported scaling function $\phi(x, y)$ :

$$
\phi(x, y)=\sum_{k_{1}, k_{2}} h_{k_{1}, k_{2}} \phi\left(2 x-k_{1}, 2 y-k_{2}\right)
$$

We call the matrix $H$ the low-pass filter which extracts the smooth parts of the image. Meanwhile, we call $G^{\mu}$ the high-pass filter for $\mu=1, \ldots, N$ which contains the detail parts of the image. Each component of the matrix $G^{\mu}$ is from the two-scale relation of the wavelet frame generators $\psi^{\mu}$ such that

$$
\psi^{\mu}(x, y)=\sum_{k_{1}, k_{2}} g_{k_{1}, k_{2}}^{\mu} \phi\left(2 x-k_{1}, 2 y-k_{2}\right), \quad \mu=1, \ldots, N
$$

Then the image decomposition is the matrix convolution of each $H$ and $G^{\mu}$ with the matrix $X_{j}$ accompanying by down sampling, which is a procedure deleting all the even number of rows and columns. Let $X_{j+1}$ be the down sampled matrix after we take convolution of $H$ with $X_{j}$ and $Y_{j+1}^{\mu}$ be the down sampled matrix after the convolution of $G^{\mu}$ with $X_{j}$. Then, the size of matrices $X_{j+1}, Y_{j+1}^{1}, \ldots, Y_{j+1}^{N}$ is a half of the size of the matrix $X_{j}$ for each $j \in \mathbf{Z}$. We call $X_{j+1}$ a low-pass sub-image and the $Y_{j+1}^{\mu}$ 's high-pass sub-images. The lowpass sub-image has low-pass (smooth) parts of the image and the high-pass subimages have high frequency (detail) parts of the image. 
The reconstruction process is the reverse process of decomposition. We insert zero rows and columns to the even number of rows and columns of matrices $X_{j+1}, Y_{j+1}^{1}, \ldots, Y_{j+1}^{N}$ respectively.

\subsection{Edge Detection}

Our wavelet frame method for edge detection can be described as in the following consecutive steps.

(i) Decomposing the original image $\mathbf{f}$, by using (13), into one low-pass sub image $\mathbf{c}_{\mathbf{1}}$ and several high-pass sub images $\mathbf{d}_{1}, \ldots, \mathbf{d}_{r}$.

(ii) Reconstructing the image by using (15), but without the low-pass component:

$$
\mathbf{f}=\mathbf{d}_{1} \oplus \cdots \oplus \mathbf{d}_{r} .
$$

As we mentioned at the beginning of this section, since the abrupt changes and detail parts of the images are located in high-pass components, the reconstructed image without low-pass component shows edges with noise. This is the noise contained in the original image.

(iii) Removing the noise after we reconstruct the image to have clear edges:

$$
\mathbf{f}=D(\mathbf{f}) \text {, }
$$

where $D$ is a Donoho's denoising operator (see [8]).

(iv) Normalizing the reconstructed image into the standard grey level from 0 to 255 and use a threshold to divide the pixel values into two major groups. That is, if a pixel value is bigger than the threshold, it is set to be 255; otherwise, it is set to be zero.

(v) Removing all isolated pixel values.

We use the tight wavelet frames based on the box spline $\phi_{2211}$ in [4] for edge detection. The box spline $\phi_{2211}$ has one low-pass filter and 8 high-pass filters.

To compare the visual effectiveness of edge detection, we also use wavelet method edge detection by using tensor products of Daubechies wavelet D6 and three commercial edge detection methods from MATLAB Image Processing Toolbox, namely Sobel, Prewitt, and Zero-crossing methods. For box spline tight wavelet frame, we choose the best edge representation among three levels of decompositions to present here. Tensor products of Daubechies wavelet have three high-pass filters each of which detect edges of horizontal, vertical, and diagonal direction respectively. Thus they have already had a disadvantage of detecting curvy edges. Among the images, tight wavelet frame detects edge more effectively in all images. Especially, it detects edge better than the 
MATLAB commercial edge detection methods in many images. We present the results visually in Figure 3 to Figure 8.

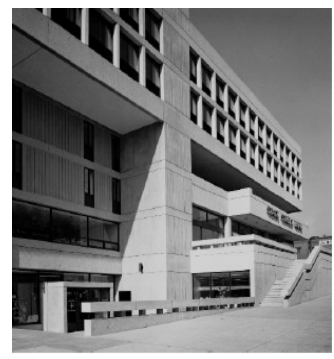

Original image

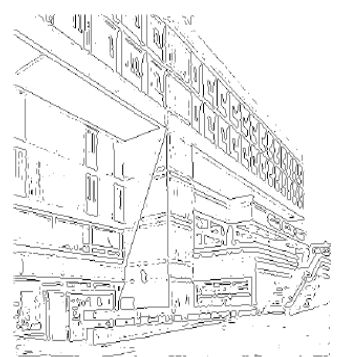

Zero Crossing

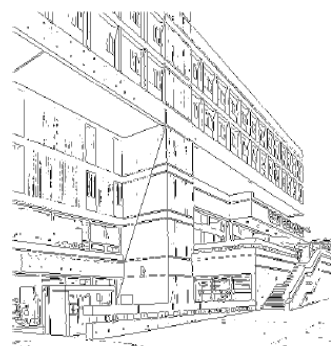

Prewitt

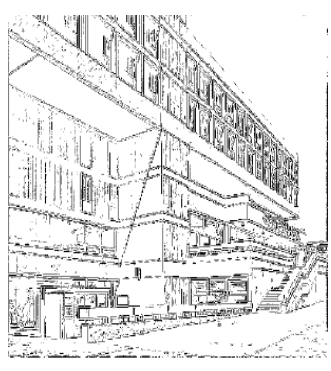

Wavelet D6

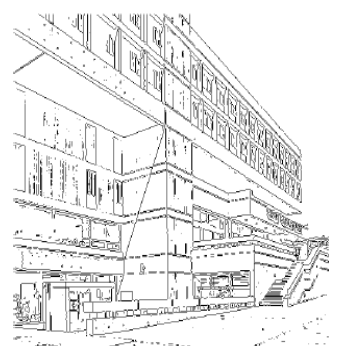

Sobel

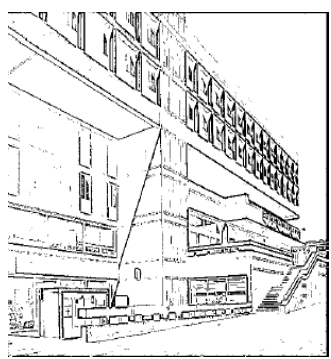

TWFD

Figure 3 Edge detection for Image Bank using 5 different methods.

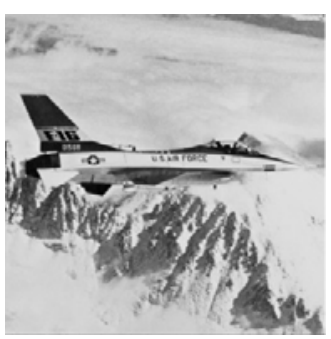

Original image

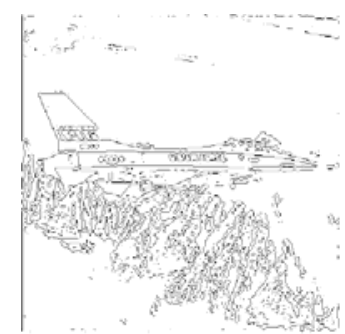

Zero Crossing

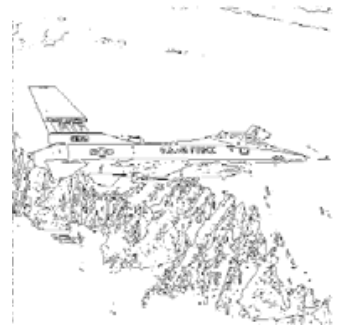

Prewitt

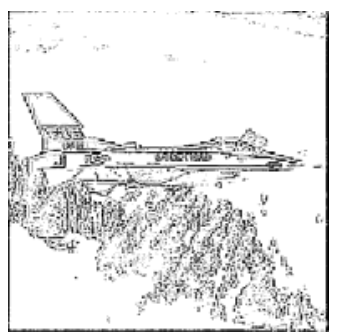

Wavelet D6

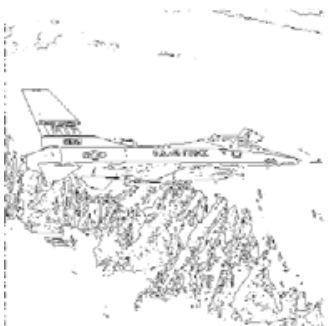

Sobel

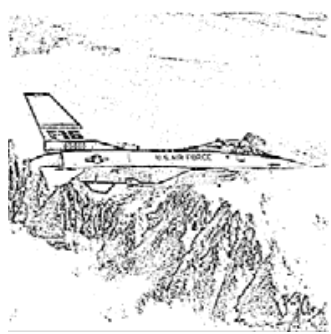

TWFD

Figure 4 Edge detection for Image F16 using 5 different methods. 


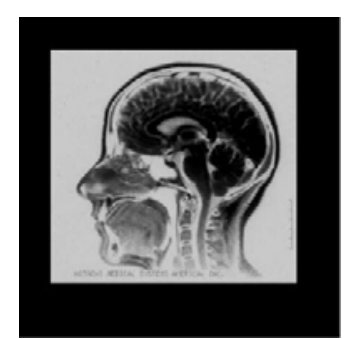

Original image

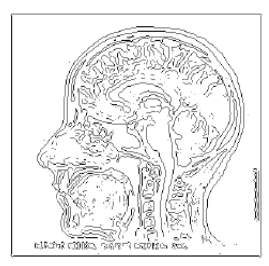

Zero Crossing

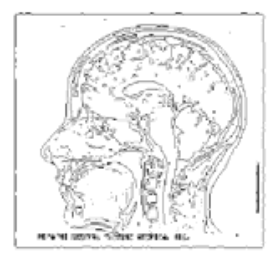

Prewitt

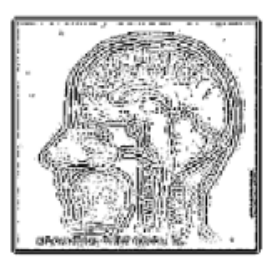

Wavelet D6

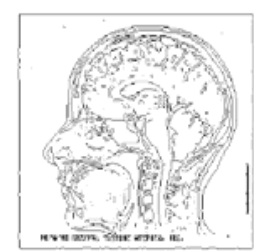

Sobel

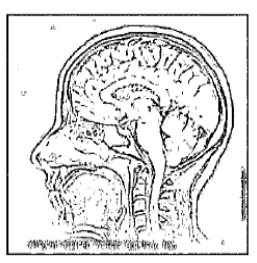

TWFD

Figure 5 Edge detection for Image Head MRI using 5 different methods.

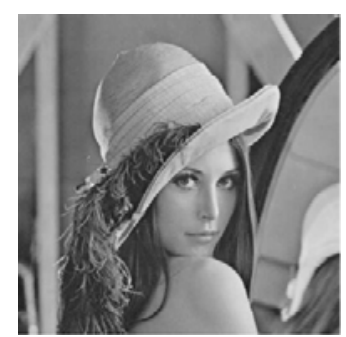

Original image

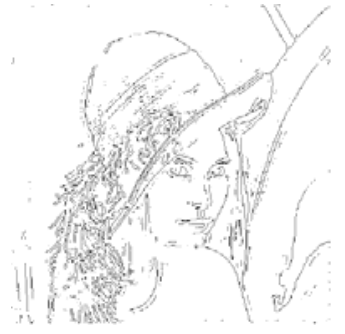

Zero Crossing

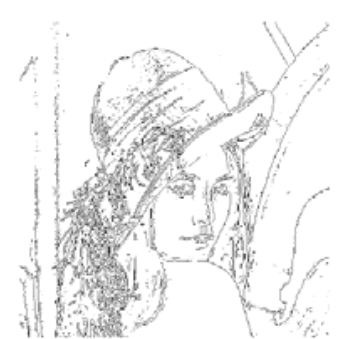

Prewitt

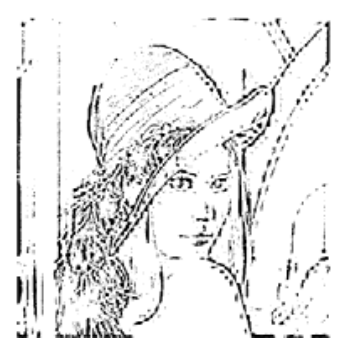

Wavelet D6

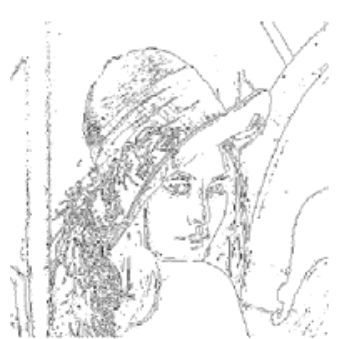

Sobel

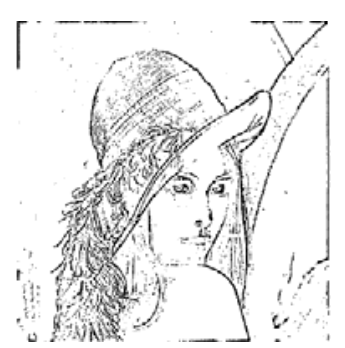

TWFD

Figure 6 Edge detection for Image Lena using 5 different methods. 


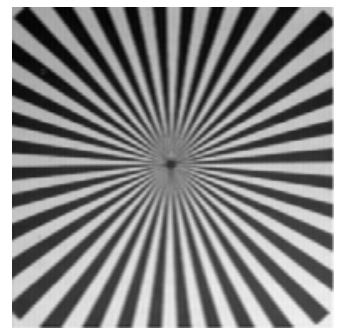

Original image

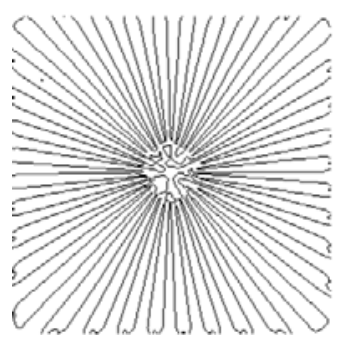

Zero Crossing

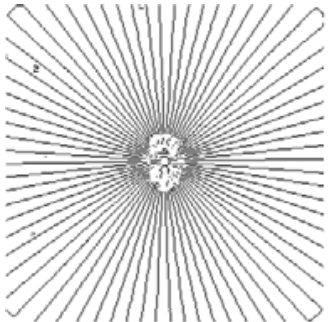

Prewitt

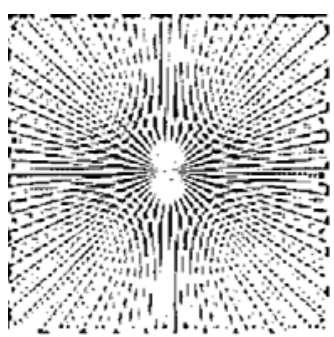

Wavelet D6

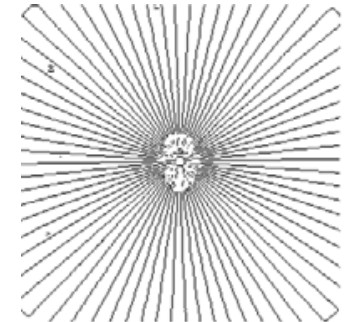

Sobel

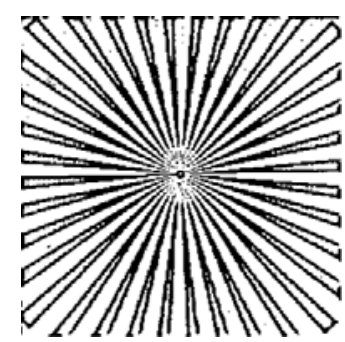

TWFD

Figure 7 Edge detection for Image Partition using 5 different methods.

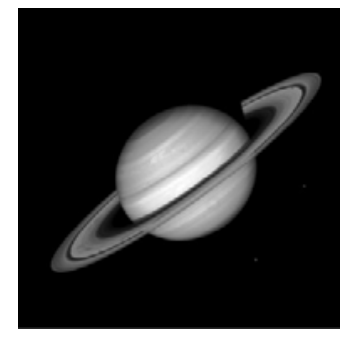

Original image

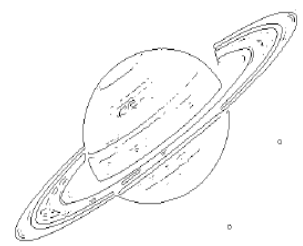

Zero Crossing

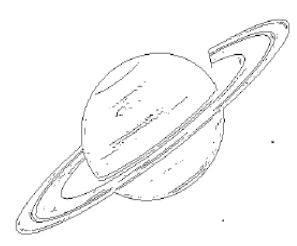

Prewitt

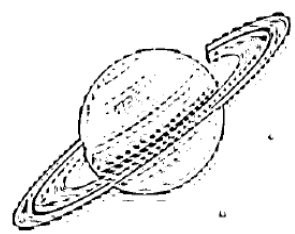

Wavelet D6

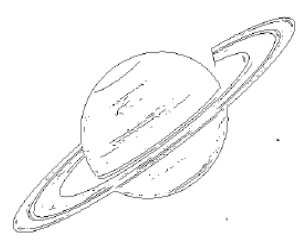

Sobel

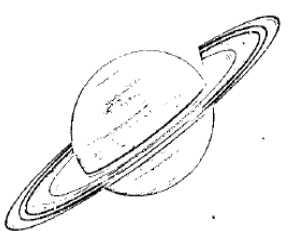

TWFD

Figure 8 Edge detection for Image Saturn using 5 different methods. 
According to the results presented visually above, we note some superiority of the application of tight wavelet frame decomposition (TWFD) in edge detection compared with the other four methods. In general, TWFD affords sharper and smoother edges for all cases of the test images. For an image with linearly parts (Figure 3), TWFD results sharp and detailed shape of edge more than the other four methods. Especially for images containing curvy parts, TWFD shows the most readable shape of edge (Figure 4, 5, 6, and 8). In addition, TWFD detects edges of patterned image clearly (Figure 7) without generating a new pattern within the resulting image.

\subsection{Image Denoising}

In our numerical experiment on image denoising, we make a noisy image first by adding a white noise to the pixel values $x_{i, j}$ of the image. The white noise has a Gaussian distribution $\delta_{i, j}$ with mean zero and variance $\sigma$. Then the pixel values $f_{i, j}$ of noisy image can be presented as

$$
f_{i, j}=x_{i, j}+\sigma \delta_{i, j}
$$

with $\delta_{i, j} \sim N(0,1)$ where $\sigma$ is chosen from values 5, 10, 15 and 20. We then decompose the image into a low-pass part and several high-pass parts of the image by using wavelets or tight wavelet frames.

Next, we apply the soft-thresholding to each high-pass part of the image. Finally, we reconstruct the image after we use noise softening. In this step, we choose optimal threshold value by using the method introduced by Donoho [8].

To measure the quality of the denoised image, we use the peak signal to noise ratio (PSNR) computed by the following formula. For a given image $x_{i, j}$ of size $N \times N$ pixels and a reconstructed image $x_{i, j}^{\prime}$, where $0 \leq x_{i, j} \leq 255$,

$$
\mathbf{P S N R}=10 \log _{10} \frac{255 N^{2}}{\sum_{i, j=1}^{N}\left(x_{i, j}-x_{i, j}^{\prime}\right)^{2}}
$$

For image denoising, we use tight wavelet frame based on the box spline $\phi_{1111}$ in [4]. The other box spline tight wavelet frames in [4] give similar results. Comparing wavelets for denoising, tight wavelet frames give better results in the set test images we tested. 
Since the most of the noise appears in the high-pass parts of the first level of decomposition, we consider only the first level of decomposition. In this case, for each given image and fixed noise we are able to find the optimal threshold values of soft-thresholding (see [8] for the detail) for the sub-images decomposed by Haar, Daubechies, or biorthogonal wavelets and wavelet frame.

We present four tables of average value of PSNR from 100 experiments according to each $\sigma$ value. Each entry in the tables contains PSNR values of noisy images and their denosied images reconstructed by tensor products of Haar, Daubechies, biorthogonal wavelets and tight wavelet frame. The largest PSNR values in Table 1 to Table 4 (typed in shaded cell) indicate the best denoised image. We see that wavelet frame has the most of largest PSNR values.

Table 1 The PSNR comparison for $\sigma=5$.

\begin{tabular}{lccccc}
\hline \multicolumn{1}{c}{ Image } & Noisy & Haar & Daubechies & Biorthogonal & TWFD \\
\hline Bank & 34.27 & $\mathbf{3 6 . 1 2}$ & 36.06 & 36.06 & 35.97 \\
F-16 & 34.16 & 36.09 & 36.45 & 36.49 & $\mathbf{3 6 . 5 0}$ \\
Head MRI & 35.29 & 37.89 & 38.47 & $\mathbf{3 8 . 7 8}$ & 38.45 \\
Lena & 34.16 & 36.06 & 36.55 & 36.63 & $\mathbf{3 6 . 6 7}$ \\
Partition & 36.11 & 35.01 & 37.04 & 37.77 & $\mathbf{3 7 . 9 8}$ \\
Saturn & 36.33 & 40.00 & 41.40 & 41.40 & $\mathbf{4 3 . 1 4}$ \\
\hline
\end{tabular}

It can be seen from Table 1, that decomposition by using Haar wavelet gives the best denoised image for image Bank with low noise level. In this case, the image Bank contains linearly parts, and Haar wavelet is known to be very good for handling such kind of images. The other methods render a low-level noisy image and result an over-smooth denoised image; without the exception of TWFD.

Table 2 The PSNR comparison for $\sigma=10$.

\begin{tabular}{lccccc}
\hline \multicolumn{1}{c}{ Image } & Noisy & Haar & Daubechies & Biorthogonal & TWFD \\
\hline Bank & 28.31 & 31.16 & 31.15 & 31.16 & $\mathbf{3 1 . 3 2}$ \\
F-16 & 28.16 & 31.20 & 31.69 & 31.76 & $\mathbf{3 2 . 0 5}$ \\
Head MRI & 29.31 & 32.91 & 33.56 & 33.84 & $\mathbf{3 4 . 1 7}$ \\
Lena & 28.15 & 31.43 & 32.07 & 32.18 & $\mathbf{3 2 . 7 8}$ \\
Partition & 30.42 & 31.90 & 32.78 & 32.74 & $\mathbf{3 3 . 0 4}$ \\
Saturn & 30.33 & 35.02 & 35.92 & 36.01 & $\mathbf{3 9 . 3 1}$ \\
\hline
\end{tabular}


For higher noise level, TWFD indicates much better denoised image even for image Bank. For curvy image (Saturn), TWFD results a highly significant PSNR improvement.

Table 3 The PSNR comparison for $\sigma=15$.

\begin{tabular}{lccccc}
\hline \multicolumn{1}{c}{ Image } & Noisy & Haar & Daubechies & Biorthogonal & TWFD \\
\hline Bank & 24.64 & 28.26 & 28.32 & 28.33 & $\mathbf{2 8 . 8 0}$ \\
F-16 & 24.66 & 28.35 & 28.89 & 28.96 & $\mathbf{2 9 . 7 4}$ \\
Head MRI & 25.82 & 30.03 & 30.56 & 30.77 & $\mathbf{3 1 . 8 4}$ \\
Lena & 24.63 & 28.75 & 29.33 & 29.41 & $\mathbf{3 0 . 8 2}$ \\
Partition & 25.24 & 27.54 & 31.21 & 30.76 & $\mathbf{3 1 . 7 8}$ \\
Saturn & 26.82 & 32.03 & 32.62 & 32.66 & $\mathbf{3 7 . 1 6}$ \\
\hline
\end{tabular}

Table 3 still shows the expected results. TWFD delivers the best quality of denoised image among the other methods, especially for totally curvy image (Saturn).

Table 4 The PSNR comparison for $\sigma=20$.

\begin{tabular}{lccccc}
\hline \multicolumn{1}{c}{ Image } & Noisy & Haar & Daubechies & Biorthogonal & TWFD \\
\hline Bank & 22.38 & 26.20 & 26.31 & 26.31 & $\mathbf{2 7 . 1 8}$ \\
F-16 & 22.24 & 26.32 & 26.87 & 26.90 & $\mathbf{2 8 . 2 3}$ \\
Head MRI & 23.36 & 27.96 & 28.35 & 28.50 & $\mathbf{3 0 . 1 5}$ \\
Lena & 22.14 & 26.80 & 27.27 & 27.34 & $\mathbf{2 9 . 5 5}$ \\
Partition & 24.33 & 26.82 & 28.72 & 28.21 & $\mathbf{3 0 . 0 0}$ \\
Saturn & 24.35 & 29.82 & 30.22 & 30.22 & $\mathbf{3 5 . 5 4}$ \\
\hline
\end{tabular}

Again from Table 4, we can see that TWFD yields the best denoised image reconstructed from quite high-level noisy image. Also, the highest PSNR improvement is achieved for the curviest image.

\section{$4 \quad$ Concluding Remark}

We know that edge detection is only a small part of an image processing, so that we consider only the visual result. We find that the edge detection method using wavelet frame decomposition (and reconstruction) provides the best result. Here, we get the most clear curvy image through the wavelet frame decomposition as this decomposition is done directly --- without doing any tensor product of one dimensional process.

It should be noticed that Donoho in [8] proved theoretically the superiority of using wavelet decomposition in image denoising. At that time, wavelet frames have not been well-developed. However, according to the results presented in 
Table 1 to Table 4, in general, the image denoising using wavelet frame decomposition gives a better result compare to that of classical wavelets decomposition.

For further work, we might apply our fast algorithm to the image processing by using the other wavelet frames. We might also take into account the algorithm complexity, including the time complexity as well as the usage of computer memory.

\section{Acknowledgement}

The authors would like to thank Dr. Armein Z. R. Langi for his valuable comments and constructive suggestions on the earlier version of this paper.

\section{References}

[1] Christensen, O., Recent Developments in Frame Theory, in Modern Mathematical Models, Methods and Algorithms for Real World Systems, A.H. Siddiqi, I.S. Duff and O. Christensen, eds., Anamaya Publishers, New Delhi, 159-195, 2006.

[2] Ron, A. \& Shen, Z., Affine Systems in $L^{2}\left(\mathbf{R}^{d}\right)$ : Analysis of the Analysis Operator, J. Funct. Anal., 148, 408-447, 1997.

[3] Daubechies, I., Ten Lectures on Wavelets, CBMS-NSF Regional Conference Series in Appl. Math., SIAM, Philadelphia, 1992.

[4] Lai, M.J. \& Stöckler, J., Construction of Multivariate Compactly Supported Tight Wavelet Frames, J. Appl. Comput. Harmonic Anal., 21, 324-348, 2006.

[5] Skopina, M., Multivariate Wavelet Frame, Tech. Report, Dept. of App. Math. and Control Processes, Saint Petersburg State University, 2007.

[6] Chui, C. K. \& He, W., Compactly Supported Tight Frames Associated with Refineble Functions, J. Appl. Comput. Harmonic Anal., 8, 293-319, 2000.

[7] Christensen, O., An Introduction to Frames and Riesz Bases, Birkhauser, Boston, 2003.

[8] Donoho, D.L., De-Noising by Soft-Thresholding, IEEE Trans. Info. Theory, 43, 933-936, 1993. 